\title{
Uracil within DNA: an actor of antiviral immunity Joséphine Sire ${ }^{1}$, Gilles Quérat ${ }^{1}$, Cécile Esnault ${ }^{2}$ and Stéphane Priet*3
}

Address: ${ }^{1}$ UMR IRD-190, Emergence des Pathologies Virales, Faculté de Médecine, 27 Bd Jean Moulin, 13005 Marseille, France, ${ }^{2}$ Unité des Rétrovirus Endogènes et Eléments Rétroïdes des Eucaryotes Supérieurs, UMR 8122 CNRS, Institut Gustave Roussy, 94805 Villejuif, France and ${ }^{3}$ Architecture et Fonction des Macromolécules Biologiques, CNRS UMR 6098, ESIL case 925, 13288 Marseille Cedex 9, France

Email: Joséphine Sire - jsire@marseille.inserm.fr; Gilles Quérat - gquerat@marseille.inserm.fr; Cécile Esnault - cesnault@igr.fr; Stéphane Priet* - stephane.priet@afmb.univ-mrs.fr

* Corresponding author

Published: 5 June 2008

Retrovirology 2008, 5:45 doi:10.1 186/1742-4690-5-45

This article is available from: http://www.retrovirology.com/content/5/I/45

(c) 2008 Sire et al; licensee BioMed Central Ltd.

This is an Open Access article distributed under the terms of the Creative Commons Attribution License (http://creativecommons.org/licenses/by/2.0), which permits unrestricted use, distribution, and reproduction in any medium, provided the original work is properly cited.
Received: 13 February 2008

Accepted: 5 June 2008

\begin{abstract}
Uracil is a natural base of RNA but may appear in DNA through two different pathways including cytosine deamination or misincorporation of deoxyuridine 5'-triphosphate nucleotide (dUTP) during DNA replication and constitutes one of the most frequent DNA lesions. In cellular organisms, such lesions are faithfully cleared out through several universal DNA repair mechanisms, thus preventing genome injury. However, several recent studies have brought some pieces of evidence that introduction of uracil bases in viral genomic DNA intermediates during genome replication might be a way of innate immune defence against some viruses. As part of countermeasures, numerous viruses have developed powerful strategies to prevent emergence of uracilated viral genomes and/or to eliminate uracils already incorporated into DNA. This review will present the current knowledge about the cellular and viral countermeasures against uracils in DNA and the implications of these uracils as weapons against viruses.
\end{abstract}

\section{Background}

Uracils in DNA may arise either from incorporation of dUTP in place of thymidine 5 '-triphosphate (dTTP) or from the generation of uracils in DNA consecutive to spontaneous or enzymatic deaminations of cytosines which, if unrepaired, will lead to non-mutagenic U:A or mutagenic U:G mispairs, respectively. Although U:A mispairs resulting from excess of cellular dUTP pool levels are not mutagenic per se, they elicit a cycle of dUMP incorporation into DNA followed by the removal of uracil base by cellular uracil DNA glycosylases (UNG) and reincorporation of dUMP during the synthesis phase. The end point of this process is the appearance of strand breaks and the loss of DNA integrity. In nonproliferating cells such as macrophages, quiescent lymphocytes or neurons the intracellular deoxynucleotide pool is low and imbalanced, with high levels of dUTP, due to the limited expres- sion of the deoxyuridine 5'-triphosphatase nucleotide hydrolase (dUTPase) that otherwise controls the dUTP/ dTTP ratio. Consequently, viruses that replicate in this adverse cellular context have a high probability to incorporate dUTP in their genome during viral replication. They have thus acquired strategies consisting in concentrating dUTPase or UNG activities in close proximity to their replication machinery. Most often they have done so by encoding themselves viral dUTPase and/or UNG in order to compensate for the low levels of these cellular enzymes. In the following we will focus on the different ways by which uracils are introduced into cellular and viral DNA and on the resulting biological consequences when uracils remain unrepaired, with a special attention to HIV-1 lentivirus. HIV-1 replicates in nondividing cells but does not encode dUTPase nor UNG. However, HIV-1 fights the detrimental uracilation of its genome induced 
by members of the APOBEC family, which are cytosine deaminases able to convert cytosine to uracil residues, through the Vif protein. Vif impedes the packaging of APOBEC members avoiding excessive G-to-A hypermutations within viral genome. The role in virus life cycle of the host-derived UNG (UNG2) enzyme that is packaged into HIV-1 virions will be discussed.

\section{Uracils in cellular or viral DNA may derive from different sources}

The common RNA base uracil (U) that is substituted by thymine (T) in DNA is able to naturally pair with adenine (A) but can also mispair with guanine $(\mathrm{G})$. The U:A pair in DNA results from the incorporation of dUTP by polymerases and constitutes a non-mutagenic event per se that can nonetheless alters promoters functions [1]. However, U:A pair may be a cytotoxic lesion or even become a mutagenic event when chromosomal abasic sites (APsites) are generated after the removal of uracils by cellular repair mechanisms [2]. The U:G mispair is a non-blocking DNA replication lesion and occurs after the deamination of a cytosine to uracil. This lesion is mutagenic, leading to a G-to-A transition mutation in one of the two daughter strands after DNA replication.

The incorporation of dUTP into DNA during replication has been estimated to be up to $10^{4}$ uracil residues in human genome per day [3] and represents the major source of uracils in DNA [4]. In eukaryotic cells, dUTP is synthesized from the phosphorylation of dUDP arising either from UDP under the action of the ribonucleoside diphosphate (rNDP) reductase or from the phosphorylation of dUMP, which is an essential intermediate for the synthesis of the intracellular dTTP pool and therefore constitutes a permanent source of dUTP (Fig. 1). DNA polymerases from eukaryotes, prokaryotes and viruses are not able to discriminate dUTP from dTTP. Thus the incorporation of dUTP directly depends on its intracellular concentration. Under physiological conditions, the concentration of dUTP and dTTP in the cell have been estimated to be $\sim 0.2 \mu \mathrm{M}$ and $37 \pm 30 \mu \mathrm{M}$, respectively [5] meaning that the normal intracellular dUTP/dTTP ratio is below or close to $1 \%$. However, some cell types such as HT29 cell line, primary spleen cells, macrophages or quiescent lymphocytes display significantly higher dUTP levels that can even exceed those of dTTP [6-8].

The deamination of cytosine residues to uracil residues in DNA can arise either from a spontaneous (non-enzymatic) or an enzymatic process. Spontaneous deamination is a frequent event that has been estimated by chemical measurements and genetic assays to occur between 70 to 200 times per cell per day [9]. In addition to cytosine deaminases, the mammalian genome encodes two distinct enzymes able to convert cytosine to uracil, namely the (cytosine-5)-methyltransferase and the APOBEC cytidine deaminase. The (cytosine-5)-methyltransferase, is in charge of the conversion of cytosines within $\mathrm{CpG}$ islets to 5-methylcytosines. In mammalian cells, 5-methylcytosines represent about 2 to 7\% of cytosines and constitute a regulatory system for transcription and can confer epigenetic informations [10]. The conversion starts with the formation of a covalent bond between the enzyme and the cytosine, leading to a transient dihydropyrimidine intermediate product that is quickly subjected to spontaneous deamination. The enzyme next catalyzes the transfer of a methyl group to the cytosine. This latter reaction uses the S-adenosylmethionine (SAM) molecule as a methyl donor. Thus, a cytosine deamination to uracil may occur in the case of the abortive catalysis by (cytosine-5)-methyltransferase [11] or in the presence of a low cellular concentration of SAM [12].

The APOBEC cytidine deaminase family members are able to deaminate cytosines within DNA and/or RNA molecules. The first member of this family, APOBEC1 (apolipoprotein B mRNA editing catalytic subunit 1), has been identified as the enzyme responsible for the tissuespecific deamination of the $\mathrm{C}^{6666}$ of the apolipoprotein $\mathrm{B}$ mRNA, leading to a premature stop codon and the expression of a truncated form of the apolipoprotein B lipidtransport protein in gastrointestinal tissues $[13,14]$. The APOBEC1 protein acts exclusively as a RNA-editing enzyme in the small intestine (where it is exclusively expressed) but can, however, deaminate cytosines present in chromosomal DNA of living bacteria $[15,16]$. These results drew attention to the possibility that APOBEC proteins could deaminate either RNA or DNA under different cellular conditions $[15,16]$. Other members of the APOBEC cytidine deaminase family, including AID (activation-induced cytidine deaminase), APOBEC2, the APOBEC3 sub-family and APOBEC4, have next been discovered and the biological function of several of them has been studied. At this time, no function has yet been attributed to APOBEC2 and APOBEC4 proteins. The AID protein, whose expression is restricted to activated mature $B$ cells, has been identified as a key factor of antibody diversification [17]. AID is required to deaminate specifically some cytosines in ssDNA of variable and switch regions of the Ig gene locus, allowing somatic hypermutation (SHM) and the class-switch recombination (CSR) processes that are needed to generate antibody diversity in response to antigens [18-21]. The APOBEC3 sub-family has been discovered when human APOBEC3G (hA3G) was reported as a host cell restriction factor for HIV replication [22]. Subsequently, it has been reported that seven APOBEC3 proteins, so-called APOBEC $3 \mathrm{~A}, 3 \mathrm{~B}, 3 \mathrm{C}, 3 \mathrm{DE}$ (the 3D and $3 \mathrm{E}$ genes encode the $\mathrm{N}$ - and $\mathrm{C}$-terminal domains of the 3DE protein, respectively), 3F, 3G and $3 \mathrm{H}$, are encoded by 

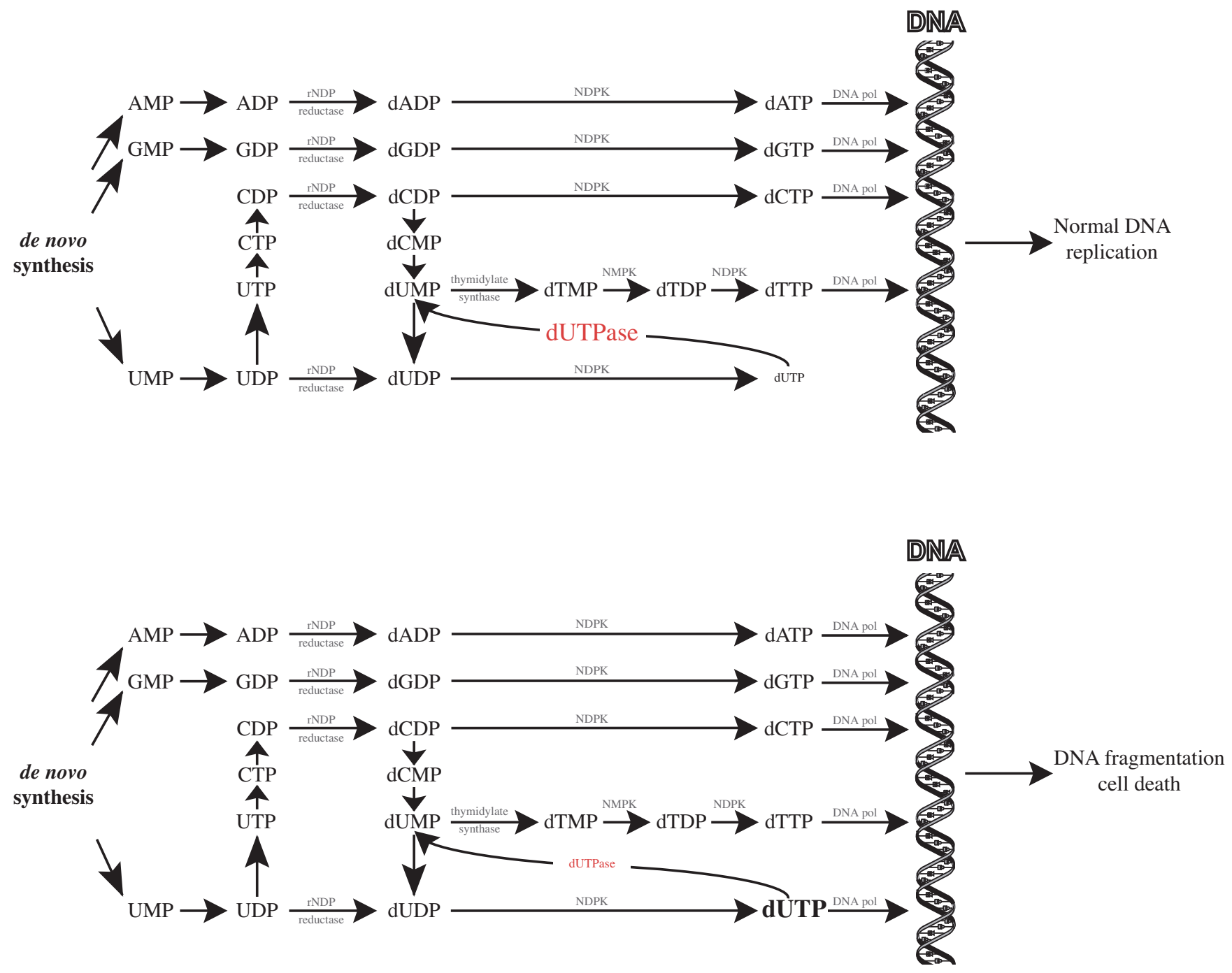

Figure I

Biosynthesis pathways of ribonucleotides and deoxyribonucleotides in mammalian cells and the possible consequence of the misincorporation and repair of uracil residues in DNA. De novo synthesis of AMP, CMP, GMP and UMP ribonucleotides allows the formation of dATP, dCTP, JGTP, dTTP and dUTP deoxyribonucleotides, which can be readily incorporated in DNA by cellular DNA polymerases. Note that dTTP derives from dUTP hydrolysis. Abbreviations: A, adenine; C, cytosine; G, guanine; T, thymine; U, uracil; MP, monophosphate; DP, diphosphate; TP, triphosphate; rNDP, ribonucleotide diphosphate; NMPK, nucleotide monophosphate kinase; NDPK, nucleotide diphosphate kinase.

the human genome $[23,24]$. These APOBEC3 proteins, with the exception of APOBEC3H, have been shown to exhibit antiviral effects against a variety of viruses, including numerous retroviruses such as HIV, SIV, MLV, HTLV and foamy viruses, hepatitis B virus and adeno-associated virus (AAV) (reviewed in [25]) (Fig. 2). The absence of antiviral effect of human APOBEC3H, in contrast to its Old World monkey (OWM) counterpart, may be explained by a poor expression [26]. The antiviral effect displayed by other human APOBEC3 proteins, with the exception of APOBEC3A, was associated with numerous cytosine deaminations (known as "editing") within the viral cDNA leading to lethal G-to-A mutations (reviewed in [25]). Indeed, APOBEC3A has been found to exert antiviral effects without cytosine deaminations although recent reports showed that it was capable of editing in vitro on single-stranded DNA [27] and on the CDNA of the avian alpharetrovirus RSV thereby inhibiting its infectivity [28]. Studies of deaminase-defective APOBEC3 mutants have shown that APOBEC3G and APOBEC3F contain antiviral determinants that can act independently of the editing process $[29,30]$. However, a recent study reported 


\begin{tabular}{|c|c|c|c|c|c|c|c|c|c|c|c|}
\hline \multirow[b]{4}{*}{ Human APOBEC3 } & \multicolumn{11}{|c|}{ Exogenous viruses } \\
\hline & \multicolumn{9}{|c|}{ Retroviruses } & \multicolumn{2}{|c|}{ Others } \\
\hline & \multicolumn{2}{|c|}{ HIV-1 } & \multirow{2}{*}{$\begin{array}{c}\text { HIV-2 } \\
\Delta V i f\end{array}$} & \multirow{2}{*}{$\begin{array}{c}\text { SIV-1 } \\
\Delta V i f\end{array}$} & \multirow[t]{2}{*}{ HTLV } & \multirow[t]{2}{*}{ MLV } & \multirow[t]{2}{*}{ EIAV } & \multirow[t]{2}{*}{ PFV } & \multirow[t]{2}{*}{ RSV } & \multirow[t]{2}{*}{$\mathrm{HBV}$} & \multirow[t]{2}{*}{ AAV } \\
\hline & WT & $\Delta V i f$ & & & & & & & & & \\
\hline $3 \mathrm{~A}$ & & - & & & & & & & $(+)$ & & + \\
\hline $3 \mathrm{~B}$ & + & + & & + & + & $(+)$ & & & + & + & - \\
\hline $3 \mathrm{C}$ & & $(+)$ & & + & + & - & & + & $(+)$ & + & - \\
\hline $3 \mathrm{DE}$ & & + & & + & + & - & & & & & \\
\hline $3 \mathrm{~F}$ & & + & & + & + & - & & + & + & + & - \\
\hline $3 \mathrm{G}$ & $(+)$ & + & + & + & + & + & + & + & + & + & - \\
\hline $3 \mathrm{H}$ & & - & & & & - & & & & & \\
\hline
\end{tabular}

Endogenous retroelements

\begin{tabular}{|c|c|c|c|c|c|}
\hline \multirow[b]{2}{*}{ Human APOBEC3 } & \multicolumn{3}{|c|}{ LTR-retrotransposons } & \multicolumn{2}{|c|}{ non-LTR retrotransposons } \\
\hline & IAP & MusD & Ty1 & L1 & Alu \\
\hline $3 \mathrm{~A}$ & + & + & + & + & + \\
\hline $3 \mathrm{~B}$ & + & $-?$ & & + & + \\
\hline $3 \mathrm{C}$ & + & $-?$ & + & + & + \\
\hline $3 \mathrm{DE}$ & & & & - & \\
\hline $3 \mathrm{~F}$ & + & + & + & $+?$ & - \\
\hline $3 \mathrm{G}$ & + & + & + & - & + \\
\hline $3 \mathrm{H}$ & & & & - & \\
\hline
\end{tabular}

+ , antiviral effect

$(+)$, modest antiviral effect

,- no effect

?, contradictory results

Figure 2

APOBEC 3 family members and their associated roles in exogenous viruses and endogenous retroelements restriction. Data are compiled from $[27,77,87,90$, I26-140].

that this previously described antiviral effect of the deaminase-defective APOBEC3G mutants was negligible as compared to the wild-type protein when equal amounts of these proteins were packaged into viral particles [31]. Beside the antiviral function of APOBEC3 proteins against exogenous viruses, some inhibitory effects have been reported on intracellular targets like the IAP, MusD or Ty1 Long Terminal Repeat (LTR)-retrotransposons and LINE1 or $A l u$ non-LTR retrotransposons through a general deaminase-dependent mechanism (again the with exception of APOBEC3A) [25]. Accordingly, several studies support the notion that one of the cellular functions of APOBEC3 proteins could be to prevent the propagation of mobile elements in their host genomes. In a general way, uracils coming from the action of the AID or the APOBEC3 proteins appear to be central actors in the adaptive or innate immune response, respectively.

\section{Genomic uracils are highly controlled in cells}

Eukaryotic and prokaryotic cells have evolved in setting up two mechanisms to impede the presence of uracils in DNA, bringing to light the highly deleterious effects of genomic uracils if unrepaired. The first mechanism in place prevents the incorporation of dUTP by acting directly on the intracellular pool of dUTP through the action of dUTPase, while the second is responsible for the 
excision of uracil once present in DNA through an universal DNA repair process called base excision repair (BER) and the use of enzymes known as uracil-DNA glycosylases (UNGs).

The dUTPase is a ubiquitous enzyme that is well conserved in all organisms. This protein maintains a low level of intracellular dUTP by converting dUTP to dUMP and inorganic pyrophosphate and also allows the biosynthesis of nucleotides derived from thymidine [32] (Fig. 1). The human dUTPase gene encodes, through alternative splicing, two isoforms that localize to either the mitochondrion or nucleus [33]. The expression of the nuclear form is cell-cycle regulated with a high expression in the $S$ phase of dividing cells that contrasts to a nearly undetectable expression in differentiated and non-dividing cells [34]. Although no human dUTPase deficiency has been observed, the absence of the dUTPase activity in prokaryotes and S. cerevisiae has demonstrated its necessity for cell viability. In addition, partial deficiency leads to enhanced frequency of spontaneous mutations, recombinations and DNA fragmentation [35-39].

The BER process is one of the cellular DNA repair mechanisms responsible for correcting most of common forms of DNA damage including the removing of genomic uracils. The DNA repair process has been extensively subjected to reviews [40] and will be shortly introduced here. It involves the recognition and the excision of an inappropriate base by a specific DNA glycosylase leading to an abasic site (AP-site) that is further cleaved on its 5' side by an apurinic/apyrimidinic (AP)-endonuclease APE, leaving a free 3 '-OH end and a 5'-deoxyribose phosphate (dRP) group. The dRP group is then incised on its 3 ' side via the lyase activity (dRPase) of the DNA polymerase $\beta$ (pol $\beta$ ) for the short patch repair pathway while a short oligonucleotide is cleaved by the flap endonuclease 1 (FEN1) for the long patch repair pathway. Finally, the resulting gap is then filled in by pol $\beta$ and/or pol $\delta / \varepsilon$ and sealed by the DNA ligase I or III. The DNA glycosylases responsible for the excision of uracils are highly conserved enzymes expressed in mammals, bacteria, yeast, or herpes- and poxviruses. In humans, several enzymes with UNG activity have been described, namely TDG, MBD4, SMUG1, UNG1 and UNG2 [41]. The UNG2 and SMUG1 enzymes have been reported as the major enzymes proficient for removing deaminated cytosines although SMUG1 is thought to act as a backup of UNG2 [42-44]. UNG2 has been also reported as the unique enzyme able to perform the excision of uracil from dUTP misincorporation $[42,43]$. The UNG1 and UNG2 enzymes are mitochondrial and nuclear isoforms, respectively, generated by alternative splicing of the human ung gene [45]. Like the dUTPase, the expression of the nuclear UNG2 form depends on the cell cycle with high levels in the S phase of dividing cells, and barely detectable levels in differentiated and non-dividing cells [34]. In contrast to UNG2 that accumulates in replication foci $[42,43]$, SMUG1 is only expressed in nucleoli where it may have a specialized role [44]. UNG-deficiency in mice and in humans leads to increased accumulation of genomic uracils, confirming its primary role in the removal of uracil from DNA [46-48].

Although UNG2 is considered to display an antimutagenic function, it seems to have an essential role in the antibody diversification process. The vast repertoire of antibody molecules, which is essential to detect and fight pathogens, is generated thanks to profound genomic changes at the Ig locus in B cells. This process occurs through numerous somatic hypermutations (SHM) that lead to the affinity maturation of antibodies, and through class-switch recombination (CSR) allowing these high affinity antibodies to gain some effector functions and to be disseminated across the body. These mechanisms are initiated from the targeted deamination of cytosines triggered by AID within the Ig locus [18-21]. The AID-generated uracils lesions are then recognized and excised by UNG2 enzyme. Replication across the resulting AP-sites by REV1 and other translesion polymerases results in the generation of transition mutations at C:G pairs $[49,50]$. Moreover, some mutations at A:T pairs can also be observed during the SHM process but the molecular mechanisms involved remain to be fully understood. The MSH2-MSH6 mismatch repair proteins could however recognize $\mathrm{U}: \mathrm{G}$ mispairs and could be required to generate A:T mutations [51-54]. A recent study has indicated that the MSH2-MSH6 heterodimer could prevent the error-free BER commonly initiated by UNG2 and that UNG2 could recruit pol $\eta$, which appears to be the sole contributor of A:T mutations [55]. Thus, UNG2 plays a key role in SHM as well as CSR processes. Indeed, UNG2-deficient humans cannot ensure CSR and therefore have elevated IgM amounts and dramatically lowered IgG, IgA and IgE levels [46]. However, the role of the uracil excision activity of UNG2 in this process seems not clear and warrants further studies. Altogether, these data highlight the crucial role of UNG2 in the adaptative immunity against numerous pathogens, like bacteria or viruses.

\section{Numerous viruses have evolved strategies to counteract uracils}

Viruses belonging to the Herpesviridae, Poxviridae and Retroviridae families have evolved in encoding their own dUTPase and/or UNG proteins, supporting the idea that these viruses are sensitive to the presence of uracil residues in their genome.

The Herpesviridae family contains members that replicate their dsDNA genome in the nucleus of a variety of cell types, including some non-dividing cells, such as neurons. 
The viral dUTPase and UNG of alpha herpesviruses, like herpes simplex virus (HSV) or varicella zoster virus (VZV), are not essential in proliferating cells [56-58] but become critical in non-dividing cells or in an in vivo murine system $[59,60]$. Beta herpesviruses, like cytomegalovirus (CMV), do not encode a functional dUTPase [61] but express their own UNG that appears to be required in non-dividing cells to compensate for the very low level of cellular UNG2 [62,63]. The gamma herpesviruses, like EpsteinBarr viruses (EBV), Kaposi's sarcoma-associated herpesviruses (HHV-8) or murine $\gamma$-herpesvirus-68 (MHV-68), also encode a functional UNG and dUTPase $[64,65]$. The EBV-encoded UNG was shown to ensure the fidelity of viral DNA replication and to promote efficient production of viral DNA [64] and the EBV-encoded dUTPase expressed by MHV-68 was found to play an important role in acute infection in the lung [66].

The members of the Poxviridae family, such as vaccinia virus, replicate their dsDNA exclusively in the cytoplasm of infected cells. Thereby, due to the total absence of cytosolic UNG activity, the inactivation of the virallyencoded UNG leads to an impaired viral replication even in proliferating cells $[67,68]$. These data highlight that uracils in the genome of DNA viruses can impair their replication and depict the necessity for these viruses to encode their own dUTPase and/or UNG enzymes to compensate for the lack of cellular counterpart during their life cycle either in non-dividing cells or in the cytoplasmic compartment.

Several members of the Retroviridae family, such as $\beta$-retroviruses including Mazon-Pfizer monkey virus (MPMV) or murine mammary tumor virus (MMTV), nonprimate lentiviruses including the ungulate lentiviruses maedivisna virus (MVV) of sheep, the caprine arthritis-encephalitis virus (CAEV), the equine infectious anemia virus (EIAV), the feline immunodeficiency virus (FIV) and the rabbit endogenous lentivirus type K (RELIK), and even many endogenous retroviruses including HERV-L, MERV$\mathrm{L}$ and HERV-K, also encode their own dUTPase protein. Interestingly, the dUTPase domain of these retroviruses can be differently located in their genome, either upstream of protease, or in-between reverse transcriptase and integrase, or downstream of integrase, indicating that retroviruses have captured a dUTPase gene during independent occasions. This suggests that the need of the dUTP hydrolysis activity is crucial to confer a selective advantage for retroviral replication. The virally-encoded dUTPase protein is expressed as a part of the Gag-pol polyprotein precursor and is expected to be encapsidated into viral particles in order to be in close spatial proximity of the reverse transcriptase [69]. Although the role of $\beta$-retroviral or endogenous retroviral dUTPases still remains to be fully determined, the study of the dUTPase of nonpri- mate lentiviruses revealed that it is required for efficient replication only in non-dividing cells, [70-74]. It is noteworthy that other retroviruses members, such as murine leukemia virus (MLV) related viruses or avian leukemia virus (ALV) related viruses, do not encode dUTPase. One can notice that they replicate their genome only in actively dividing cells, which may explain why they do not need to encode dUTPase.

Collectively, these data show that (i) although not mutagenic per se, uracils in viral genomes coming from dUTP misincorporation alter viral replication, (ii) high dUTP levels are present in the nucleus of non-dividing cells or in the cytoplasm of proliferating cells that express low levels of dUTPase and UNG enzymes, and finally (iii) uracils in viral DNA genome have to be highly controlled to allow an efficient viral replication.

\section{APOBEC3 proteins as a part of the innate anti-HIV-I immunity and viral countermeasures}

Since Sheehy et al. discovered that the human endogenous APOBEC3G protein acts as a restriction factor to inhibit HIV-1 replication [22], the question of innate immunity against viruses has gained much attention. From that time, several other APOBEC3 proteins, namely APOBEC3F [75,76], -3B [77,78] and -3DE [79] have been shown to exert antiviral effects against HIV-1. This restriction effect requires the packaging of APOBEC3 proteins into virions budding from HIV-1 infected cells. Studies on the incorporation of these antiviral factors into viral particles brought conflicting evidences leading to propose that either a specific interaction with the nucleocapsid (NC) domain of Gag polyprotein [80-82], or an association with viral or cellular RNA [83-86] were required for the encapsidation. Recently, studies with highly divergent Gag polyproteins [87] or RNase A treatment $[83,84,88]$ brought more evidence to the second model where APOBEC3 packaging involves an RNA intermediate. Following the entry of APOBEC3G-containing viral particles in target cells, virion-associated APOBEC3 proteins trigger extensive cytosine deaminations to uracils in neosynthesized viral minus-strand DNA [89-92]. This process occurs through a target site specificity depending on the nature of the APOBEC3 protein. Thus, APOBEC3G prefers to deaminate the dinucleotide $\mathrm{C} C$ (underline marks the target) on minus-strand CDNA [89-92], while APOBEC3F or -3B

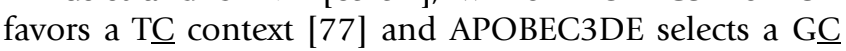
context [79]. This event takes place next to the degradation of the RNA template by the RNase $\mathrm{H}$ activity of RT because (i) the APOBEC3G proteins act only on singlestranded DNA [93] and (ii) the APOBEC3G activity is inhibited by viral genomic RNA [94]. The deamination of cytosine by APOBEC3 proteins leads to lethal G-to-A hypermutations in viral plus-strand cDNA [89-92,95]. However, in several in vitro experimental assays, some 
APOBEC3 family members are depicted as able to restrict some retroviruses, retroelements or other viruses through mechanisms independent of DNA deaminations (reviewed in [25]) (Fig. 2). In the case of APOBEC3G, it has been reported that it exhibits both deaminasedependent and deaminase-independent antiviral activities. The deaminase-independent activity could be due to directly inhibiting the strand transfer or the processivity of reverse transcriptase $[96,97]$.

To counteract the editing process and to protect its genome from G-to-A hypermutations, HIV-1 evolved in encoding the Vif protein that exhibits the property to bind APOBEC3G and to induce its proteasomal degradation, thus depleting APOBEC3G at the site of virion budding [98-100]. The degradation of APOBEC3G requires the formation of a Vif-Cul5-elongins B and C-Rbx1 complex, which allows the polyubiquitination of APOBEC3G. It has also been reported that Vif could interfere with APOBEC3G translation [101] or directly with its packaging [102]. Interestingly, the Vif-mediated degradation appears to be more potent on APOBEC3G than on APOBEC $3 \mathrm{~F}$ or $-3 \mathrm{DE}$ and has not been observed for APOBEC3B [98-100]. In addition, the aspartate residue at position 128 of APOBEC3G from different species has been reported to drive the interaction with Vif and thus to govern species specificity [102-106]. Indeed, HIV-1 Vif protein was efficient to bind to and inhibit the packaging of human APOBEC3G but not that of the simian (African green monkey or rhesus monkey) or murine counterparts $[102,107]$.

Collectively, these data show that cells take advantage of uracil susceptibility displayed by numerous viruses to inhibit their propagation through the expression of the APOBEC3 family members. Unfortunately, HIV-1 illustrates the high capacity of viruses to evolve rapidly and to develop mechanisms to thwart this innate antiviral immunity.

\section{Primate HIV-I lentivirus controls uracils within viral DNA}

Lentiviruses are divided into nonprimate and primate subgroups and share common characteristics, such as the ability to infect non-dividing cells and to replicate their genome in the cytoplasm of infected cells. In this cellular context, nonprimate lentiviruses control the deleterious uracilation of their genome through the expression of a virally-encoded dUTPase. The analysis of primate lentivirus genomes reveals that they do not encode dUTPase nor UNG-like enzymes. Using a yeast two-hybrid screen, cellular UNG2 was shown to associate with the accessory HIV-1 Vpr protein [108] and to be incorporated into HIV1 viral particles [109-114]. Although Vpr was initially suspected to play a role in the packaging of UNG2 $[110,111]$, it was later demonstrated by three other groups that
UNG2 packaging was independent of the presence of Vpr in viral particles $[109,112,113,115,116]$. Indeed, the integrase (IN) domain in the context of the HIV-1 Gag-Pol precursor was identified as the sole determinant to target UNG2 into virions and we have demonstrated that the leucine 172 residue of IN was critical for UNG2 encapsidation $[109,115]$. The UNG2 packaging was specific to HIV-1, as neither HIV-2 nor simian immunodeficiency virus (SIV) packaged UNG2 [116]. It remains to be determined whether the latter package another cellular UNG or whether they manage to circumvent the uracilation of their genomes through other means.

Although it has been shown that $\mathrm{Vpr}$ could participate in the fidelity of reverse transcriptase [110], the role of the Vpr-UNG2 association remains to be evaluated. Interestingly, recent results showed that the expression of Vpr in B-cells had a dominant-negative effect on the class switch recombination (CSR) events [117]. This effect appeared to be dependent on the interaction of Vpr with the WxxF motif contained in UNG2 suggesting that Vpr could compete with some endogenous Vpr-like factors important for UNG function in CSR. Indeed, the CSR is initiated by AID and the AID-induced uracils are removed by a UNG2- or a MSH2-dependent mechanism [118], leading to a not fully characterized dsDNA cleavage step required for CSR (reviewed in [119]). By analogy, it is possible that the VprUNG2 complex may play a role in inhibiting dsDNA cleavages subsequent to the UNG2-induced removal of uracils in viral cDNA. This assumption may explain why the infectivity of HIV-1 virions that contain small amounts of APOBEC-induced uracils was increased by Vpr [113] and why Vpr seems to be important for viral replication in non-dividing cells [111]. Future experiments demonstrating a direct implication of the Vpr-UNG complex in the protection of dsDNA from cleavage are awaited. Another point that deserves future investigations concerns the fact that $\mathrm{Vpr}$ could potentially target hAPOBEC3G, which contains in its sequence two WxxF motifs. If such a Vpr-hAPOBEC3G interaction exists, it could assign an additional role of $\mathrm{Vpr}$ as a back-up of Vif.

The role of the virion-associated UNG2 in the viral life cycle was evaluated by our laboratory through the specific depletion of UNG2 by RNA interference-mediated knockdown and through the inhibition of UNG2 catalytic activity by the expression of the uracil-DNA glycosylase inhibitor (Ugi) in virus producing cells [120]. It was found that HIV-1 viruses produced from dividing as well as nondividing UNG2-deficient cells were noninfectious, demonstrating that UNG2 within the viral particle is essential for infectivity. This also demonstrated that the depletion of UNG2 in viral particles could not be compensated by endogenous UNG2 present in target cells, suggesting that the spatial proximity of UNG2 and the replication 
enzymes in the viral nucleocapsid is important. In addition, the targeting of the CAEV dUTPase into UNG2-deficient viral particles was able to rescue the viral infectivity, pointing out that dUTP misincorporation could be at the origin of a lethal reverse transcription process. Indeed, HIV-1 lysates contained all necessary factors to repair uracils originating from dUTP misincorporation during the reverse transcription process $[120,121]$. These data pointed out that, to prevent dUTP misincorporations, HIV-1 is endowed with an original uracil BER pathway that is initiated by UNG2 and associated with a previously unknown dNTP-dependent AP-endonuclease activity of reverse transcriptase (RT) [120]. Although others [110] and we [121] failed to identify the human AP-endonuclease APE1 within HIV-1 particles, a recent report indicated that APE1 molecules could be detected in viral particles [114]. The presence of APE1 within viral particles could participate in association with UNG2 and RT to the repair of dUTP misincorporations and could thus have a redundant function in HIV-1 particles. Meanwhile, the main role of APE1 within viral particles seems rather to be the degradation of APOBEC3G-edited nascent HIV-1 DNA after the excision of uracils by UNG2 [114], suggesting a specialized function of UNG2 and APE1 hijacked by the cell to amplify the anti-HIV-1 effect of APOBEC3G in the absence of Vif. It should be mentioned that the degradation of newly synthesized transcripts cannot be performed by the dNTP-dependent AP-endonuclease activity of RT because (i) the RT can cleave UNG2-induced AP-sites only during the reverse transcription step, i. e. when a template is available (be it RNA or DNA) [120,122], (ii) the substrate for APOBEC3G is the ssDNA $[93,95]$ and, (iii) the degradation of the viral RNA template by the RNase $\mathrm{H}$ activity of RT, which inhibits the APOBEC3G activity [94], is a prerequisite for the cytosine deamination process $[93,95]$.

Our data demonstrating that UNG2 was necessary for viral infectivity are in sharp contrast with data from two other groups $[112,114]$ showing that UNG2 was somewhat dispensable for HIV-1 replication. Kaiser and Emerman reported that, although UNG2 was efficiently encapsidated into HIV-1 virions, it played no role in infectivity, neither for wild type (WT) viruses nor for Vif-minus viruses in the presence of APOBEC3G [112]. In contrast, Yang's group has reported that UNG2 encapsidation from producer cells, while dispensable for replication of WT viruses, was involved in the rescue of Vif-minus HIV-1 in the presence of APOBEC3G [114]. Although all groups target UNG2 inhibition or depletion (by the use of Ugi as an inhibitor of UNG2 activity, UNG2 specific siRNA or UNG2-/- mutants cells), it is difficult to reconcile these conflicting data given that methodologies differ. However, two drawbacks have not been addressed. First, the quasi-irreversibility of the binding of Ugi to UNG has been demonstrated for pure E. Coli UDG $[123,124]$ and not for human UNG2 either pure or already engaged in its interaction with HIV-1 integrase (IN). Therefore it remains possible that the incoming Ugi associated to the virally-encapsidated UNG2 may well dissociate by competition with the vast excess of free UNG2 in the recipient cells, leading to a functional UNG2-positive entering replication complex. Second, the EBV transformed B cell lines from $U N G^{-/}$patients may well express high levels of EBVencoded dUTPase and/or UNG. Thus, the possibility exists that EBV-encoded UNG could be packaged into HIV-1 particles and compensates for the lack of human UNG2 in the $U_{N G}-1$ cell line. This hypothesis might be verified by analyzing the EBV-transformed cell line for the expression of EBV-encoded UNG and its packaging to HIV-1. In addition, the expression of EBV-encoded dUTPase will be sufficient to abrogate the cytoplasmic dUTP pool levels. Therefore, the best approach to definitely conclude on the role of UNG2 in HIV-1 infectivity would be the use of primary $\mathrm{T}$ lymphocyte or macrophages, instead of EBV-transformed B cell lines, derived from $U N G^{-}-$patients and would thus avoid some manipulations of cells including overexpression of Ugi or RNA interference. In addition, one can try to make his mind about the role of UNG2 in the HIV-1 life cycle by some considerations of evolutionary perspectives. Yang's data point to a positive role of UNG2 packaging only when Vif is deficient, but are neutral for a WT virus. It is difficult to pinpoint any evolutionary forces, which could select for UNG2 packaging in such a case. The Kaiser and Emerman data are consistent with an accidental packaging of UNG2, an epiphenomenon not related with any evolutionary advantage. Such hypothesis would explain the lack of UNG2 packaging into HIV-2 and SIV virions, two closely related cousins of HIV-1, although no one has still looked at whether other cellular UNGs might be encapsidated. In contradiction with that hypothesis is the presence in all other non-primate lentiviruses of an enzymatic activity, so-called dUTPase, in charge of preventing uracilation of their genome. Given that all lentiviruses infect non-dividing macrophagic and dendritic cells as their main and common target cells, it is tempting to speculate that they acquired means to use enzymatic activities enhancing their replication in the adverse context of nondividing cells. This hypothesis is conforted by the fact that an ancestral dUTPase gene was identified in the HIV-1 envelop gene [125].

Taken together, these data sustained the notion that the presence of uracils in viral reverse transcripts is a constant threat for HIV-1 replication. The virus consequently evolved in developing strategies including both the expression of the virally-encoded Vif protein to inhibit the packaging of host-derived cytosine deaminase APOBEC proteins and the packaging of host-encoded uracil DNA 
glycosylase UNG2 to initiate uracil repair of misincorporated dUTP.

\section{Concluding remarks}

To date, considerable progress has been made in understanding the interactions between superior organisms and their pathogens. The discovery of the APOBEC family members has highlighted a central role for uracils as a barrier against infectious agents, firstly as an actor of antibody diversification in adaptative immunity, and secondly as a potent antiviral per se. This latter feature has been particularly well documented in the case of infection by HIV-1 and revealed how this virus faces up to the uracilation of its genome. During the natural course of HIV-1 infection, the Vif expression level in infected cells seems to be sufficient to avoid extensive APOBEC3Gmediated cytosine deamination of the viral genome that otherwise will lead to error catastrophe. It remains possible however that some APOBEC3G proteins escape the Vif-mediated degradation. This in turn could help in the emergence of G-to-A mutations enhancing drug resistance and immune escape processes of HIV-1. Likewise, UNG2 encapsidation level within viral particles seems to be sufficient to prevent the dUTP incorporation in viral reverse transcripts. However, nothing is known about the efficiency of HIV-1 replication when infected cells exhibit high levels of intracellular dUTP. Therefore, it may be interesting to test whether enhancing artificially the dUTP pool levels in HIV-1 infected cells may represent an alternative approach to antiviral therapy. This could be done by inhibition of thymidylate synthase or dUTPase. The development of drugs against these enzymatic activities may deserve to be evaluated as a viable therapeutic approach.

\section{List of abbreviations}

dUTP: deoxyuridine 5'-triphosphate nucleotide; AID: activation-induced cytidine deaminase; APOBEC: apolipoprotein B mRNA editing catalytic polypeptide; UNG: uracil DNA glycosylase; dUTPase: deoxyuridine 5'-triphosphatase nucleotide hydrolase; BER: base excision repair; SHM: somatic hypermutation; CSR: class-switch recombination; HIV-1: immunodeficiency virus type-1; Vif: viral infectivity factor.

\section{Competing interests}

The authors declare that they have no competing interests.

\section{Authors' contributions}

JS, GQ, CE and SP participating equally in revising the intellectual content and drafting of the manuscript. Author(s) read and approved the final manuscript.

\section{Acknowledgements}

This work was supported by INSERM, by CNRS, by Ensemble Contre le SIDA (Sidaction) and by the French Agency against AIDS (ANRS).

\section{References}

I. Verri A, Mazzarello P, Biamonti G, Spadari S, Focher F: The specific binding of nuclear protein(s) to the CAMP responsive element (CRE) sequence (TGACGTCA) is reduced by the misincorporation of $\mathbf{U}$ and increased by the deamination of $\mathbf{C}$. Nucleic Acids Res 1990, I 8:5775-5780.

2. Auerbach P, Bennett RA, Bailey EA, Krokan HE, Demple B: Mutagenic specificity of endogenously generated abasic sites in Saccharomyces cerevisiae chromosomal DNA. Proc Natl Acad Sci USA 2005, 102:17711-17716.

3. Mosbaugh DW, Bennett SE: Uracil-excision DNA repair. Prog Nucleic Acid Res Mol Biol 1994, 48:3 I 5-370.

4. Andersen S, Heine T, Sneve R, Konig I, Krokan HE, Epe B, Nilsen H: Incorporation of dUMP into DNA is a major source of spontaneous DNA damage, while excision of uracil is not required for cytotoxicity of fluoropyrimidines in mouse embryonic fibroblasts. Carcinogenesis 2005, 26:547-555.

5. Traut TW: Physiological concentrations of purines and pyrimidines. Mol Cell Biochem 1994, I 40: I-22.

6. Cross DR, Miller BJ, James SJ: A simplified HPLC method for simultaneously quantifying ribonucleotides and deoxyribonucleotides in cell extracts or frozen tissues. Cell Prolif 1993, 26:327-336.

7. Horowitz RW, Zhang H, Schwartz EL, Ladner RD, Wadler S: Measurement of deoxyuridine triphosphate and thymidine triphosphate in the extracts of thymidylate synthase-inhibited cells using a modified DNA polymerase assay. Biochem Pharmacol 1997, 54:635-638.

8. Aquaro S, Calio R, Balzarini J, Bellocchi MC, Garaci E, Perno CF: Macrophages and HIV infection: therapeutical approaches toward this strategic virus reservoir. Antiviral Res 2002, 55:209-225.

9. Kavli B, Otterlei M, Slupphaug G, Krokan HE: Uracil in DNA - general mutagen, but normal intermediate in acquired immunity. DNA Repair (Amst) 2007, 6:505-5I6.

10. Reik W, Dean W, Walter J: Epigenetic reprogramming in mammalian development. Science 200I, 293: 1089-1093.

II. Seifarth W, Krieg-Schneider F, Skladny H, Marachelian A, Germaier $H$, Hehlmann R, Leib-Mosch C: Impact of human endogenous retroviral elements on cellular genes: strategy for isolation of LTR-driven chimeric transcripts. Leukemia I999, I 3:S97-98.

12. Shen JC, Rideout WM 3rd, Jones PA: High frequency mutagenesis by a DNA methyltransferase. Cell 1992, 71:1073-1080.

13. Powell LM, Wallis SC, Pease RJ, Edwards YH, Knott TJ, Scott J: A novel form of tissue-specific RNA processing produces apolipoprotein-B48 in intestine. Cell 1987, 50:831-840.

14. Teng B, Burant CF, Davidson NO: Molecular cloning of an apolipoprotein B messenger RNA editing protein. Science 1993, 260:1816-1819.

15. Harris RS, Petersen-Mahrt SK, Neuberger MS: RNA editing enzyme APOBECI and some of its homologs can act as DNA mutators. Mol Cell 2002, I 0: I247-1253.

16. Beale RC, Petersen-Mahrt SK, Watt IN, Harris RS, Rada C, Neuberger MS: Comparison of the differential context-dependence of DNA deamination by APOBEC enzymes: correlation with mutation spectra in vivo. J Mol Biol 2004, 337:585-596.

17. Muramatsu M, Sankaranand VS, Anant S, Sugai M, Kinoshita K, Davidson NO, Honjo T: Specific expression of activation-induced cytidine deaminase (AID), a novel member of the RNA-editing deaminase family in germinal center B cells. J Biol Chem 1999, 274: 18470-18476.

18. Muramatsu M, Kinoshita K, Fagarasan S, Yamada S, Shinkai Y, Honjo $\mathrm{T}$ : Class switch recombination and hypermutation require activation-induced cytidine deaminase (AID), a potential RNA editing enzyme. Cell 2000, 102:553-563.

19. Revy P, Muto T, Levy Y, Geissmann F, Plebani A, Sanal O, Catalan N, Forveille M, Dufourcq-Labelouse R, Gennery A, et al.: Activationinduced cytidine deaminase (AID) deficiency causes the autosomal recessive form of the Hyper-IgM syndrome (HIGM2). Cell 2000, 102:565-575.

20. Dickerson SK, Market E, Besmer E, Papavasiliou FN: AID mediates hypermutation by deaminating single stranded DNA. J Exp Med 2003, I 97:1291-1296.

21. Bransteitter R, Pham P, Scharff MD, Goodman MF: Activationinduced cytidine deaminase deaminates deoxycytidine on 
single-stranded DNA but requires the action of RNase. Proc Natl Acad Sci USA 2003, 100:4102-4I07.

22. Sheehy AM, Gaddis NC, Choi JD, Malim MH: Isolation of a human gene that inhibits HIV-I infection and is suppressed by the viral Vif protein. Nature 2002, 4I 8:646-650.

23. Sawyer SL, Emerman M, Malik HS: Ancient adaptive evolution of the primate antiviral DNA-editing enzyme APOBEC3G. PLoS Biol 2004, 2:E275.

24. Jarmuz A, Chester A, Bayliss J, Gisbourne J, Dunham I, Scott J, Navaratnam N: An anthropoid-specific locus of orphan C to U RNAediting enzymes on chromosome 22. Genomics 2002, 79:285-296.

25. Holmes RK, Malim MH, Bishop KN: APOBEC-mediated viral restriction: not simply editing? Trends Biochem Sci 2007, 32: $118-128$

26. OhAinle M, Kerns JA, Malik HS, Emerman M: Adaptive evolution and antiviral activity of the conserved mammalian cytidine deaminase APOBEC3H. J Virol 2006, 80:3853-3862.

27. Chen H, Lilley CE, Yu Q, Lee DV, Chou J, Narvaiza I, Landau NR, Weitzman MD: APOBEC3A is a potent inhibitor of adenoassociated virus and retrotransposons. Curr Biol 2006, 16:480-485.

28. Wiegand HL, Cullen BR: Inhibition of alpharetrovirus replication by a range of human APOBEC3 proteins. J Virol 2007, 8I:13694-13699.

29. Newman EN, Holmes RK, Craig HM, Klein KC, Lingappa JR, Malim $\mathrm{MH}$, Sheehy AM: Antiviral function of APOBEC3G can be dissociated from cytidine deaminase activity. Curr Biol 2005, 15:166-170.

30. Holmes RK, Koning FA, Bishop KN, Malim MH: APOBEC3F can inhibit the accumulation of HIV-I reverse transcription products in the absence of hypermutation. Comparisons with APOBEC3G. J Biol Chem 2007, 282:2587-2595.

31. Miyagi E, Opi S, Takeuchi H, Khan M, Goila-Gaur R, Kao S, Strebel K: Enzymatically active APOBEC3G is required for efficient inhibition of human immunodeficiency virus type I. J Virol 2007, 81:13346-13353.

32. Shlomai J, Kornberg A: Deoxyuridine triphosphatase of Escherichia coli. Purification, properties, and use as a reagent to reduce uracil incorporation into DNA. J Biol Chem 1978, 253:3305-3312.

33. Ladner RD, Caradonna S): The human dUTPase gene encodes both nuclear and mitochondrial isoforms. Differential expression of the isoforms and characterization of a cDNA encoding the mitochondrial species. I Biol Chem 1997, 272:19072-19080.

34. Fischer JA, Muller-Weeks S, Caradonna S: Proteolytic degradation of the nuclear isoform of uracil-DNA glycosylase occurs during the $\mathbf{S}$ phase of the cell cycle. DNA Repair (Amst) 2004, 3:505-513.

35. Tye BK, Lehman IR: Excision repair of uracil incorporated in DNA as a result of a defect in dUTPase. J Mol Biol 1977, I I 7:293-306.

36. Hochhauser SJ, Weiss B: Escherichia coli mutants deficient in deoxyuridine triphosphatase. J Bacteriol 1978, 134:157-166.

37. el-Haij $\mathrm{HH}$, Zhang $\mathrm{H}$, Weiss B: Lethality of a dut (deoxyuridine triphosphatase) mutation in Escherichia coli. J Bacteriol 1988, 170:1069-1075.

38. Gadsden MH, Mclntosh EM, Game JC, Wilson PJ, Haynes RH: dUTP pyrophosphatase is an essential enzyme in Saccharomyces cerevisiae. Embo J 1993, I 2:4425-443 I.

39. Ingraham HA, Dickey L, Goulian M: DNA fragmentation and cytotoxicity from increased cellular deoxyuridylate. Biochemistry 1986, 25:3225-3230.

40. Almeida KH, Sobol RW: A unified view of base excision repair: lesion-dependent protein complexes regulated by posttranslational modification. DNA Repair (Amst) 2007, 6:695-7II.

41. Krokan HE, Drablos F, Slupphaug G: Uracil in DNA - occurrence, consequences and repair. Oncogene 2002, $21: 8935-8948$.

42. Otterlei M, Warbrick E, Nagelhus TA, Haug T, Slupphaug G, Akbari M, Aas PA, Steinsbekk K, Bakke O, Krokan HE: Post-replicative base excision repair in replication foci. Embo J 1999, I 8:3834-3844.

43. Nilsen H, Rosewell I, Robins P, Skjelbred CF, Andersen S, Slupphaug G, Daly G, Krokan HE, Lindahl T, Barnes DE: Uracil-DNA glycosy- lase (UNG)-deficient mice reveal a primary role of the enzyme during DNA replication. Mol Cell 2000, 5:1059- 1065.

44. Kavli B, Sundheim O, Akbari M, Otterlei M, Nilsen H, Skorpen F, Aas PA, Hagen L, Krokan HE, Slupphaug G: hUNG2 is the major repair enzyme for removal of uracil from U:A matches, $U: G$ mismatches, and $U$ in single-stranded DNA, with hSMUG I as a broad specificity backup. I Biol Chem 2002, 277:39926-39936.

45. Nilsen H, Otterlei M, Haug T, Solum K, Nagelhus TA, Skorpen F, Krokan HE: Nuclear and mitochondrial uracil-DNA glycosylases are generated by alternative splicing and transcription from different positions in the UNG gene. Nucleic Acids Res 1997, 25:750-755.

46. Imai K, Slupphaug G, Lee WI, Revy P, Nonoyama S, Catalan N, Yel L, Forveille M, Kavli B, Krokan HE, et al.: Human uracil-DNA glycosylase deficiency associated with profoundly impaired immunoglobulin class-switch recombination. Nat Immunol 2003, 4:1023-1028.

47. An Q, Robins $P$, Lindahl T, Barnes DE: C -.> T mutagenesis and gamma-radiation sensitivity due to deficiency in the Smug I and Ung DNA glycosylases. Embo J 2005, 24:2205-2213.

48. Kavli B, Andersen S, Otterlei M, Liabakk NB, Imai K, Fischer A, Durandy A, Krokan HE, Slupphaug G: B cells from hyper-IgM patients carrying UNG mutations lack ability to remove uracil from ssDNA and have elevated genomic uracil. J Exp Med 2005, 201:201I-202I.

49. Simpson LJ, Sale JE: Revl is essential for DNA damage tolerance and non-templated immunoglobulin gene mutation in a vertebrate cell line. Embo / 2003, 22:1654-1664.

50. Jansen JG, Langerak P, Tsaalbi-Shtylik A, Berk P van den, Jacobs H, de Wind N: Strand-biased defect in C/G transversions in hypermutating immunoglobulin genes in Revl-deficient mice. J Exp Med 2006, 203:319-323.

51. Martin A, Li Z, Lin DP, Bardwell PD, Iglesias-Ussel MD, Edelmann W, Scharff MD: Msh2 ATPase activity is essential for somatic hypermutation at a-T basepairs and for efficient class switch recombination. J Exp Med 2003, 198: II7I-1/78.

52. Martomo SA, Yang WW, Gearhart PJ: A role for Msh6 but not Msh3 in somatic hypermutation and class switch recombination. J Exp Med 2004, 200:6I-68.

53. Rada C, Di Noia JM, Neuberger MS: Mismatch recognition and uracil excision provide complementary paths to both Ig switching and the A/T-focused phase of somatic mutation. Mol Cell 2004, 16:163-171.

54. Shen HM, Tanaka A, Bozek G, Nicolae D, Storb U: Somatic hypermutation and class switch recombination in Msh6(-/-) Ung(-/) double-knockout mice. J Immunol 2006, I77:5386-5392.

55. Delbos F, Aoufouchi S, Faili A, Weill JC, Reynaud CA: DNA polymerase eta is the sole contributor of $A / T$ modifications during immunoglobulin gene hypermutation in the mouse. $J$ Exp Med 2007, 204: 17-23.

56. Caradonna SJ, Cheng YC: Induction of uracil-DNA glycosylase and dUTP nucleotidohydrolase activity in herpes simplex virus-infected human cells. J Biol Chem I98I, 256:9834-9837.

57. Fisher FB, Preston VG: Isolation and characterisation of herpes simplex virus type I mutants which fail to induce dUTPase activity. Virology 1986, 148:190-197.

58. Reddy SM, Williams M, Cohen Jl: Expression of a uracil DNA glycosylase (UNG) inhibitor in mammalian cells: varicellazoster virus can replicate in vitro in the absence of detectable UNG activity. Virology 1998, 25 I:393-40I

59. Pyles RB, Sawtell NM, Thompson RL: Herpes simplex virus type I dUTPase mutants are attenuated for neurovirulence, neuroinvasiveness, and reactivation from latency. / Virol 1992, 66:6706-67I3.

60. Pyles RB, Thompson RL: Evidence that the herpes simplex virus type I uracil DNA glycosylase is required for efficient viral replication and latency in the murine nervous system. J Virol 1994, 68:4963-4972.

61. Caposio P, Riera L, Hahn G, Landolfo S, Gribaudo G: Evidence that the human cytomegalovirus $46-k D a$ UL72 protein is not an active dUTPase but a late protein dispensable for replication in fibroblasts. Virology 2004, 325:264-276.

62. Prichard MN, Duke GM, Mocarski ES: Human cytomegalovirus uracil DNA glycosylase is required for the normal temporal 
regulation of both DNA synthesis and viral replication. J Virol 1996, 70:3018-3025

63. Courcelle CT, Courcelle J, Prichard MN, Mocarski ES: Requirement for uracil-DNA glycosylase during the transition to latephase cytomegalovirus DNA replication. J Virol 200I, 75:7592-760I

64. Lu CC, Huang HT, Wang JT, Slupphaug G, Li TK, Wu MC, Chen YC Lee CP, Chen MR: Characterization of the uracil-DNA glycosylase activity of Epstein-Barr virus BKRF3 and its role in lytic viral DNA replication. J Virol 2007, 8 I: I I 95- I 208.

65. Kremmer E, Sommer P, Holzer D, Galetsky SA, Molochkov VA, Gurtsevitch V, Winkelmann C, Lisner R, Niedobitek G, Grasser FA: Kaposi's sarcoma-associated herpesvirus (human herpesvirus-8) ORF54 encodes a functional dUTPase expressed in the lytic replication cycle. J Gen Virol I999, 80(Pt 5): | 305-I 3 I0.

66. Song MJ, Hwang S, Wong WH, Wu TT, Lee S, Liao HI, Sun R: Identification of viral genes essential for replication of murine gamma-herpesvirus $\mathbf{6 8}$ using signature-tagged mutagenesis. Proc Natl Acad Sci USA 2005, 102:3805-3810.

67. Millns AK, Carpenter MS, DeLange AM: The vaccinia virusencoded uracil DNA glycosylase has an essential role in viral DNA replication. Virology 1994, 198:504-5I3.

68. Ellison KS, Peng W, McFadden G: Mutations in active-site residues of the uracil-DNA glycosylase encoded by vaccinia virus are incompatible with virus viability. J Virol 1996, 70:7965-7973.

69. Elder JH, Lerner DL, Hasselkus-Light CS, Fontenot DJ, Hunter E, Luciw PA, Montelaro RC, Phillips TR: Distinct subsets of retroviruses encode dUTPase. J Virol 1992, 66: I79|-1794.

70. Lichtenstein DL, Rushlow KE, Cook RF, Raabe ML, Swardson CJ, Kociba GJ, Issel CJ, Montelaro RC: Replication in vitro and in vivo of an equine infectious anemia virus mutant deficient in dUTPase activity. J Virol 1995, 69:288I-2888

7I. Threadgill DS, Steagall WK, Flaherty MT, Fuller FJ, Perry ST, Rushlow KE, Le Grice SF, Payne SL: Characterization of equine infectious anemia virus dUTPase: growth properties of a dUTPasedeficient mutant. J Virol 1993, 67:2592-2600.

72. Wagaman PC, Hasselkus-Light CS, Henson M, Lerner DL, Phillips TR, Elder JH: Molecular cloning and characterization of deoxyuridine triphosphatase from feline immunodeficiency virus (FIV). Virology 1993, 196:45|-457.

73. Steagall WK, Robek MD, Perry ST, Fuller FJ, Payne SL: Incorporation of uracil into viral DNA correlates with reduced replication of EIAV in macrophages. Virology 1995, 21 0:302-313.

74. Turelli P, Petursson G, Guiguen F, Mornex JF, Vigne R, Querat G: Replication properties of dUTPase-deficient mutants of caprine and ovine lentiviruses. J Virol 1996, 70:1213-1217.

75. Wiegand HL, Doehle BP, Bogerd HP, Cullen BR: A second human antiretroviral factor, $A P O B E C 3 F$, is suppressed by the HIVI and HIV-2 Vif proteins. Embo J 2004, 23:245I-2458.

76. Zheng YH, Irwin D, Kurosu T, Tokunaga K, Sata T, Peterlin BM: Human APOBEC3F is another host factor that blocks human immunodeficiency virus type I replication. J Virol 2004, 78:6073-6076

77. Bishop KN, Holmes RK, Sheehy AM, Davidson NO, Cho SJ, Malim $\mathrm{MH}$ : Cytidine deamination of retroviral DNA by diverse APOBEC proteins. Curr Biol 2004, 14:1392-1396.

78. Doehle BP, Schafer A, Cullen BR: Human APOBEC3B is a potent inhibitor of HIV-I infectivity and is resistant to HIV-I Vif. Virology 2005, 339:28I-288.

79. Dang $Y$, Wang $X$, Esselman $W$ J, Zheng $Y H$ : Identification of APOBEC3DE as another antiretroviral factor from the human APOBEC family. J Virol 2006, 80: 10522-10533.

80. Alce TM, Popik W: APOBEC3G is incorporated into virus-like particles by a direct interaction with HIV-I Gag nucleocapsid protein. J Biol Chem 2004, 279:34083-34086.

81. Cen S, Guo F, Niu M, Saadatmand J, Deflassieux J, Kleiman L: The interaction between HIV-I Gag and APOBEC3G. I Biol Chem 2004, 279:33I77-33I84

82. Zennou V, Perez-Caballero D, Gottlinger H, Bieniasz PD: APOBEC3G incorporation into human immunodeficiency virus type I particles. J Virol 2004, 78: I 2058- I 206I.

83. Khan MA, Kao S, Miyagi E, Takeuchi H, Goila-Gaur R, Opi S, Gipson $\mathrm{CL}$, Parslow TG, Ly H, Strebel K: Viral RNA is required for the association of APOBEC3G with human immunodeficiency virus type I nucleoprotein complexes. J Virol 2005, 79:5870-5874
84. Svarovskaia ES, Xu H, Mbisa JL, Barr R, Gorelick RJ, Ono A, Freed EO, Hu WS, Pathak VK: Human apolipoprotein B mRNA-editing enzyme-catalytic polypeptide-like 3G (APOBEC3G) is incorporated into HIV-I virions through interactions with viral and nonviral RNAs. J Biol Chem 2004, 279:35822-35828.

85. Khan MA, Goila-Gaur R, Opi S, Miyagi E, Takeuchi H, Kao S, Strebel $\mathrm{K}$ : Analysis of the contribution of cellular and viral RNA to the packaging of APOBEC3G into HIV-I virions. Retrovirology 2007, 4:48.

86. Wang T, Tian C, Zhang W, Luo K, Sarkis PT, Yu L, Liu B, Yu Y, Yu XF: 7SL RNA mediates virion packaging of the antiviral cytidine deaminase APOBEC3G. / Virol 2007, 8 I: |3 II2-13124

87. Dutko JA, Schafer A, Kenny AE, Cullen BR, Curcio MJ: Inhibition of a yeast LTR retrotransposon by human APOBEC3 cytidine deaminases. Curr Biol 2005, 15:661-666.

88. Schafer A, Bogerd HP, Cullen BR: Specific packaging of APOBEC3G into HIV-I virions is mediated by the nucleocapsid domain of the gag polyprotein precursor. Virology 2004, 328:163-168

89. Lecossier D, Bouchonnet F, Clavel F, Hance AJ: Hypermutation of HIV-I DNA in the absence of the Vif protein. Science 2003, 300: 1112

90. Mangeat B, Turelli P, Caron G, Friedli M, Perrin L, Trono D: Broad antiretroviral defence by human APOBEC3G through lethal editing of nascent reverse transcripts. Nature 2003, 424:99-103

91. Harris RS, Bishop KN, Sheehy AM, Craig HM, Petersen-Mahrt SK, Watt IN, Neuberger MS, Malim MH: DNA deamination mediates innate immunity to retroviral infection. Cell 2003, I I 3:803-809.

92. Zhang H, Yang B, Pomerantz RJ, Zhang C, Arunachalam SC, Gao L: The cytidine deaminase CEMI5 induces hypermutation in newly synthesized HIV-I DNA. Nature 2003, 424:94-98.

93. Suspene R, Sommer P, Henry M, Ferris S, Guetard D, Pochet S, Chester A, Navaratnam N, Wain-Hobson S, Vartanian JP: APOBEC3G is a single-stranded DNA cytidine deaminase and functions independently of HIV reverse transcriptase. Nucleic Acids Res 2004, 32:242I-2429.

94. Soros VB, Yonemoto W, Greene WC: Newly synthesized APOBEC3G is incorporated into HIV virions, inhibited by HIV RNA, and subsequently activated by RNase H. PLoS Pathog 2007, 3:el5.

95. Yu Q, Konig R, Pillai S, Chiles K, Kearney M, Palmer S, Richman D, Coffin JM, Landau NR: Single-strand specificity of APOBEC3G accounts for minus-strand deamination of the HIV genome. Nat Struct Mol Biol 2004, I I :435-442.

96. Iwatani Y, Chan DS, Wang F, Maynard KS, Sugiura W, Gronenborn AM, Rouzina I, Williams MC, Musier-Forsyth K, Levin JG: Deaminase-independent inhibition of HIV-I reverse transcription by APOBEC3G. Nucleic Acids Res 2007, 35:7096-7108.

97. Li XY, Guo F, Zhang L, Kleiman L, Cen S: APOBEC3G inhibits DNA strand transfer during HIV-I reverse transcription. J Biol Chem 2007, 282:32065-32074.

98. Marin M, Rose KM, Kozak SL, Kabat D: HIV-I Vif protein binds the editing enzyme APOBEC $3 G$ and induces its degradation. Nat Med 2003, 9:1398-I403.

99. Mehle A, Strack B, Ancuta P, Zhang C, McPike M, Gabuzda D: Vif overcomes the innate antiviral activity of APOBEC3G by promoting its degradation in the ubiquitin-proteasome pathway. J Biol Chem 2004, 279:7792-7798.

100. Yu X, Yu Y, Liu B, Luo K, Kong W, Mao P, Yu XF: Induction of APOBEC3G ubiquitination and degradation by an HIV-I VifCul5-SCF complex. Science 2003, 302: 1056-1060.

10I. Stopak K, de Noronha C, Yonemoto W, Greene WC: HIV-I Vif blocks the antiviral activity of APOBEC3G by impairing both its translation and intracellular stability. Mol Cell 2003, I2:59|-60|.

102. Mariani R, Chen D, Schrofelbauer B, Navarro F, Konig R, Bollman B, Munk C, Nymark-McMahon H, Landau NR: Species-specific exclusion of APOBEC3G from HIV-I virions by Vif. Cell 2003, II 4:2|-3|.

103. Bogerd HP, Doehle BP, Wiegand HL, Cullen BR: A single amino acid difference in the host $A P O B E C 3 G$ protein controls the primate species specificity of HIV type I virion infectivity factor. Proc Natl Acad Sci USA 2004, 101:3770-3774. 
104. Mangeat $B$, Turelli $P$, Liao $S$, Trono $D$ : A single amino acid determinant governs the species-specific sensitivity of APOBEC3G to Vif action. J Biol Chem 2004, 279: | 448 | - 4483.

105. Schrofelbauer $B$, Chen $D$, Landau NR: $A$ single amino acid of APOBEC 3G controls its species-specific interaction with virion infectivity factor (Vif). Proc Natl Acad Sci USA 2004, I0I:3927-3932.

106. Xu H, Svarovskaia ES, Barr R, Zhang Y, Khan MA, Strebel K, Pathak VK: A single amino acid substitution in human APOBEC3G antiretroviral enzyme confers resistance to HIV-I virion infectivity factor-induced depletion. Proc Natl Acad Sci USA 2004, 101:5652-5657.

107. Gaddis NC, Chertova E, Sheehy AM, Henderson LE, Malim MH: Comprehensive investigation of the molecular defect in vifdeficient human immunodeficiency virus type I virions. J Virol 2003, 77:5810-5820

108. Bouhamdan M, Benichou S, Rey F, Navarro JM, Agostini I, Spire B, Camonis J, Slupphaug G, Vigne R, Benarous R, Sire J: Human immunodeficiency virus type I Vpr protein binds to the uracil DNA glycosylase DNA repair enzyme. I Virol 1996, 70:697-704.

109. Willetts KE, Rey F, Agostini I, Navarro JM, Baudat Y, Vigne R, Sire J: DNA repair enzyme uracil DNA glycosylase is specifically incorporated into human immunodeficiency virus type viral particles through a Vpr-independent mechanism. J Virol 1999, 73:1682-1688.

110. Mansky LM, Preveral S, Selig L, Benarous R, Benichou S: The interaction of vpr with uracil DNA glycosylase modulates the human immunodeficiency virus type I In vivo mutation rate. J Virol 2000, 74:7039-7047.

III. Chen R, Le Rouzic E, Kearney JA, Mansky LM, Benichou S: Vprmediated incorporation of UNG2 into HIV-I particles is required to modulate the virus mutation rate and for replication in macrophages. J Biol Chem 2004, 279:284I 9-28425.

1 12. Kaiser SM, Emerman M: Uracil DNA glycosylase is dispensable for human immunodeficiency virus type I replication and does not contribute to the antiviral effects of the cytidine deaminase Apobec3G. J Virol 2006, 80:875-882.

I 13. Schrofelbauer B, Yu Q, Zeitlin SG, Landau NR: Human immunodeficiency virus type I Vpr induces the degradation of the UNG and SMUG uracil-DNA glycosylases. I Virol 2005, 79:10978-10987.

114. Yang B, Chen K, Zhang C, Huang S, Zhang H: Virion-associated uracil DNA glycosylase-2 and apuriniclapyrimidinic endonuclease are involved in the degradation of APOBEC3G-edited nascent HIV-I DNA. J Biol Chem 2007, 282: | |667-II675.

II5. Priet S, Navarro JM, Querat G, Sire J: Reversion of the Lethal Phenotype of an HIV-I Integrase Mutant Virus by Overexpression of the Same Integrase Mutant Protein. J Biol Chem 2003, 278:20724-20730

116. Priet S, Navarro JM, Gros N, Querat G, Sire J: Differential incorporation of uracil DNA glycosylase UNG2 into HIV-I, HIV-2, and SIV(MAC) viral particles. Virology 2003, 307:283-289.

117. Begum NA, Izumi N, Nishikori M, Nagaoka H, Shinkura R, Honjo T: Requirement of non-canonical activity of uracil DNA glycosylase for class switch recombination. I Biol Chem 2007 282:73।-742.

1 18. Di Noia JM, Williams GT, Chan DT, Buerstedde JM, Baldwin GS, Neuberger MS: Dependence of antibody gene diversification on uracil excision. J Exp Med 2007, 204:3209-3219.

119. Chaudhuri J, Basu U, Zarrin A, Yan C, Franco S, Perlot T, Vuong B, Wang J, Phan RT, Datta A, et al.: Evolution of the immunoglobulin heavy chain class switch recombination mechanism. Adv Immunol 2007, 94:157-2I4.

120. Priet S, Gros N, Navarro JM, Boretto J, Canard B, Querat G, Sire J: HIV-I-associated uracil DNA glycosylase activity controls dUTP misincorporation in viral DNA and is essential to the HIV-I life cycle. Mol Cell 2005, 17:479-490.

121. Priet S, Navarro JM, Gros N, Querat G, Sire J: Functional Role of HIV-I Virion-associated Uracil DNA Glycosylase 2 in the Correction of G:U Mispairs to G:C Pairs. J Biol Chem 2003, 278:4566-457।.

122. Priet S, Sire J, Querat G: Uracils as a cellular weapon against viruses and mechanisms of viral escape. Curr HIV Res 2006, 4:31-42.
123. Bennett SE, Mosbaugh DW: Characterization of the Escherichia coli uracil-DNA glycosylase.inhibitor protein complex. J Biol Chem 1992, 267:22512-2252।

124. Bennett SE, Schimerlik MI, Mosbaugh DW: Kinetics of the uracilDNA glycosylase/inhibitor protein association. Ung interaction with Ugi, nucleic acids, and uracil compounds. J Biol Chem 1993, 268:26879-26885.

125. Abergel C, Robertson DL, Claverie JM: "Hidden" dUTPase sequence in human immunodeficiency virus type I gp I20. Virol 1999, 73:75I-753.

126. Bogerd HP, Wiegand HL, Doehle BP, Lueders KK, Cullen BR: APOBEC3A and APOBEC3B are potent inhibitors of LTRretrotransposon function in human cells. Nucleic Acids Res 2006, 34:89-95

127. Bogerd HP, Wiegand HL, Hulme AE, Garcia-Perez JL, O'Shea KS, Moran JV, Cullen BR: Cellular inhibitors of long interspersed element I and Alu retrotransposition. Proc Natl Acad Sci USA 2006, 103:8780-8785

128. Esnault C, Heidmann O, Delebecque F, Dewannieux M, Ribet D, Hance AJ, Heidmann T, Schwartz O: APOBEC3G cytidine deaminase inhibits retrotransposition of endogenous retroviruses. Nature 2005, 433:430-433.

129. Esnault C, Millet J, Schwartz O, Heidmann T: Dual inhibitory effects of APOBEC family proteins on retrotransposition of mammalian endogenous retroviruses. Nucleic Acids Res 2006, 34:|522-|53|.

130. Schumacher AJ, Nissley DV, Harris RS: APOBEC3G hypermutates genomic DNA and inhibits Tyl retrotransposition in yeast. Proc Natl Acad Sci USA 2005, 102:9854-9859.

13I. Muckenfuss H, Hamdorf M, Held U, Perkovic M, Lower J, Cichutek K, Flory E, Schumann GG, Munk C: APOBEC3 proteins inhibit human LINE-I retrotransposition. J Biol Chem 2006, 28I:22I6I-22I 72 .

132. Stenglein MD, Harris RS: APOBEC3B and APOBEC3F inhibit LI retrotransposition by a DNA deamination-independent mechanism. J Biol Chem 2006, 28 I: 16837-16841.

133. Turelli P, Mangeat B, Jost S, Vianin S, Trono D: Inhibition of hepatitis B virus replication by APOBEC3G. Science 2004, 303: 1829.

134. Yu Q, Chen D, Konig R, Mariani R, Unutmaz D, Landau NR: APOBEC3B and APOBEC $3 C$ are potent inhibitors of simian immunodeficiency virus replication. I Biol Chem 2004, 279:53379-53386.

135. Baumert TF, Rosler C, Malim MH, von Weizsacker F: Hepatitis B virus DNA is subject to extensive editing by the human deaminase APOBEC3C. Hepatology 2007, 46:682-689.

136. Ribeiro AC, Maia e Silva A, Santa-Marta M, Pombo A, Moniz-Pereira J, Goncalves J, Barahona I: Functional analysis of Vif protein shows less restriction of human immunodeficiency virus type 2 by APOBEC3G. J Virol 2005, 79:823-833.

137. Russell RA, Wiegand HL, Moore MD, Schafer A, McClure MO, Cullen $B R$ : Foamy virus Bet proteins function as novel inhibitors of the APOBEC 3 family of innate antiretroviral defense factors. J Virol 2005, 79:8724-873।.

138. Delebecque F, Suspene R, Calattini S, Casartelli N, Saib A, Froment A, Wain-Hobson S, Gessain A, Vartanian JP, Schwartz O: Restriction of foamy viruses by APOBEC cytidine deaminases. I Virol 2006, 80:605-6I4.

139. Noguchi C, Hiraga N, Mori N, Tsuge M, Imamura M, Takahashi S, Fujimoto $Y$, Ochi $H$, Abe $H$, Maekawa $T$, et al:: Dual effect of APOBEC3G on Hepatitis B virus. J Gen Virol 2007, 88:432-440.

140. Suspene R, Guetard D, Henry M, Sommer P, Wain-Hobson S, Vartanian JP: Extensive editing of both hepatitis B virus DNA strands by APOBEC 3 cytidine deaminases in vitro and in vivo. Proc Natl Acad Sci USA 2005, 102:8321-8326. 